\title{
Anwendung von Pflegeprodukten und ölen in der Säuglingspflege
}

\author{
Claudia Dachs, Ulla Busmann, Hans F. Merk
}

\begin{abstract}
Die Hautpflege bei Neugeborenen und Säuglingen ist ein wichtiges Thema in der Beratung junger Eltern. Teil 3 der Artikelserie verrät, wie Öle in der Säuglingspflege sich auf die Hautschutzbarriere auswirken, welche Inhaltsstoffe in Pflegeprodukten enthalten sind und welche Empfehlungen zur Beschaffenheit von Reinigungs- und Pflegeprodukten für die Säuglingshaut auf Basis wissenschaftlicher Untersuchungen gegeben werden können.
\end{abstract}

\section{Öle in der Säuglingspflege}

Öle sind aus der Pflege von Neugeborenen und Säuglingen nicht wegzudenken. Doch welche sind besonders zu empfehlen - pflanzliche Öle oder Paraffinöle? Diese Frage wird schon lange kontrovers diskutiert. Nachfolgend seien die wichtigsten Fakten zu Eigenschaften und Wirkungen verschiedener Ölvarianten zusammengefasst. Grundsätzlich können weder für die eine noch die andere Variante pauschale Aussagen getroffen werden. Spezifische Eigenschaften, Herstellungsverfahren und Qualitäten spielen eine entscheidende Rolle.

\section{Wie Öle auf die Hautschutzbarriere wirken}

Geeignete Pflegeöle können positive Wirkungen auf die Funktion der Hautschutzbarriere entfalten. Denn sie helfen, einen erhöhten transepidermalen Wasserverlust (TEWL) zu vermeiden und die Feuchtigkeit in den obersten Hautschichten zu halten.

Babypflegeöle unterstützen die noch nicht voll ausgebildete Barrierefunktion der Säuglingshaut. Die Feuchtigkeit der Haut wird bewahrt, ohne ihre Atmung zu blockieren. Dieser sogenannte teilokklusive Effekt ist bei Pflanzen- und Paraffinölen vergleichbar. Beide dringen gleich tief in das Stratum corneum, die aus Korneozyten bestehende äußerste Schicht der Epidermis (Oberhaut) ein. Die Annahme, dass Paraffinöle zu einem vollständigen Abschluss der Haut führen, ist heute wissenschaftlich widerlegt.

Unterschiede in der Wirkung auf die Hautschutzbarriere bestehen in folgenden Punkten:

\section{Pflanzenöle}

- werden größtenteils in der Haut enzymatisch in ihre Bestandteile aufgespalten und in die Hautstruktur eingebaut. Dadurch ziehen sie besser in die Haut ein.

- enthalten zusätzliche Wirkstoffe, die positiv auf die Haut wirken können, z. B. entzündungshemmende Linolensäure oder als Radikalfänger wirkendes Vitamin E.

\section{Paraffinöle}

- werden nicht wie Pflanzenöle aufgespalten und interagieren nicht mit dem Stoffwechsel der Haut. Dadurch hält der schützende, d. h. wasserhaltende Effekt länger an.

- enthalten in ihrer reinen Form keine zusätzlichen Inhaltsstoffe. Aufgrund dieser Neutralität sind sie besonders für die Pflege von zu Allergien neigender Haut geeignet.

\section{Qualität und Sicherheit von Pflanzen- und Paraffinölen}

Wie beurteilen nun Wissenschaftler die Eignung unterschiedlicher Ölsorten für die Säuglingspflege? Die wichtigste Erkenntnis: Qualität und Sicherheit hängen wesentlich vom Herstellungsverfahren und den spezifischen Eigenschaften des Öls ab.

Grundlage aller Pflanzenöle sind pflanzliche Fettsäuren, die denen der Haut ähneln. Paraffinöle basieren ursprünglich auf Pflanzen und Mikroorganismen, die sich zu Erdöl umgewandelt haben. Daraus ergeben sich Unterschiede in Zusammensetzung, Verarbeitung und Wirkung ( Tab. 1). 
•Tab. 1 Eigenschaften von Pflanzenölen und Paraffinölen

\section{Pflanzenöle}

\section{Ursprung:}

pflanzliche Bestandteile

\section{Herstellungsverfahren:}

meist (Kalt-)Pressung, Extraktion mit Lösungsmitteln oder Raffination

\section{Qualität:}

schwankend je nach Herkunftsort, Klima, Erntezeitpunkt und Herstellungsverfahren

\section{Reinheit und Sicherheit:}

- bei Kaltpressung: höherer Gehalt an wertvollen Begleitstoffen wie Vitamin E, können aber auch Pestizidspuren oder Geruchsstoffe enthalten

- bei Raffination: weniger naturbelassen, dafür reiner bei hohem Gehalt an Ölsäure, z. B. in Olivenöl: können schon in geringen Mengen die Hautbarriere angreifen

\section{Haltbarkeit:}

- steigt, je weniger ungesättigte Fettsäuren enthalten sind

- „Ranzigwerden“ kann durch stabilisierende oder antioxidative

Zusatzstoffe wie Vitamin E verzögert werden

\section{Eignung für die Säuglingspflege:}

- nur spezielle, für Säuglingshaut ausgewiesene Öle verwenden

\section{Paraffinöle}

\section{Ursprung:}

Pflanzen und Organismen, die auf natürliche Art zu Erdöl umgewandelt wurden.

\section{Herstellungsverfahren:}

meist Destillation oder Raffination

\section{Qualität:}

gleichbleibend, unterliegen keinen natürlichen Schwankungen

\section{Reinheit und Sicherheit:}

- frei von allergenem Potenzial

- Weißöle (Paraffinum Liquidum) sind hoch gereinigt, farb- und geruchslos

- gehören zu den sichersten Stoffen in der Kosmetik

- enthalten keine Ölsäure und greifen somit die Hautbarriere nicht an

Haltbarkeit:

- sehrlang

- hohe Stabilität gegenüber Luftsauerstoff, Wasser oder Mikroorganismen

- keine Konservierungsstoffe notwendig

\section{Eignung für die Säuglingspflege:}

- auch bei zu Allergien neigender Haut einsetzbar

\section{Anwendung von Ölen in der Säuglingspflege}

Wissenschaftliche Studien haben gezeigt, dass Pflegeöle grundsätzlich für die Pflege von Säuglingshaut geeignet sind. Doch wann ist welches Öl die beste Wahl? Hier einige praktische Empfehlungen für die wichtigsten Anwendungsbereiche.

\section{Trockene Haut}

Pflanzen- und Paraffinöle erhöhen die Wasserspeicherung in den oberen Hautschichten und senken den transepidermalen Wasserverlust. Gleichzeitig schützen Öle die Säuglingshaut vor äußeren Einflüssen und unterstützen ihre Regeneration. Sie stärken somit die Hautschutzbarriere.

Differenzierung und Empfehlungen: Bei Paraffinölen hält der Schutzeffekt länger an. Unter den Pflanzenölen sind solche mit mehrfach ungesättigten Fettsäuren zu empfehlen: Linolensäure beispielsweise kann Entzündungen hemmen und die Barrierefunktion stärken.

\section{Empfindliche und zu Allergien neigende Haut}

Da Paraffinöle kein allergenes Potenzial aufweisen, sind sie uneingeschränkt anwendbar.

Differenzierung und Empfehlungen: Einige Pflanzenöle sind wegen ihres Allergiepotenzials nicht zu empfehlen, z.B. Erdnuss- und Sesamöl. Auch Pflanzenöle, die nicht lichtgeschützt, kühl und verschlossen gelagert wurden, oder Speiseöle sollten nicht verwendet werden. Außerdem sind raffinierte Öle, die weitgehend von (allergenen) Begleitstoffen befreit wurden, kaltgepressten vorzuziehen.

\section{Kopfgneis (seborrhoische Dermatitis)}

Kopfgneis lässt sich mit Ölen schonend entfernen.

Differenzierung und Empfehlungen: Pflanzen- und Paraffinöle sind hier gleichermaßen gut geeignet. Am besten Babypflegöl über Nacht einwirken lassen und am nächsten Morgen mit einem Babyshampoo gründlich auswaschen.

\section{Reinigung}

Babypflegeöle sind zur Reinigung des Windelbereichs gut geeignet, weil sie schonend wasserunlösliche Rückstände entfernen. Die teilokklusive Wirkung des Öls auf der Haut hilft, Austrocknung zu vermeiden.

Differenzierung und Empfehlungen: Pflanzenöle wirken zusätzlich rückfettend, während die hautschützende Wirkung bei Paraffinölen länger anhält. Bevor eine Creme zur möglichen Prophylaxe aufgetragen wird, sollte das Öl mit einem Tuch abgetupft werden.

\section{Bad}

Im Badewasser gehen Öle keine Verbindung mit Wasser ein, sondern schwimmen an der Oberfläche. Daher kann die Haut des Säuglings glitschig werden. Auch 
haben Öle allein im Wasser keine reinigende Wirkung. Es sollte kein Öl ohne Emulgator in das Wasser gegeben werden. Spezielle hydrophile Babybadeöle oder Babybadezusätze mit milden Reinigungsstoffen sind zu bevorzugen.

\section{Massage}

Eine sanfte Massage mit Ölen kann entspannen, beruhigen und die Eltern-Kind-Bindung fördern.

Differenzierung und Empfehlungen: Pflanzenöle ziehen besser in die Haut ein als Paraffinöle. Sie haben zusätzlichen Wirkcharakter. Für die Säuglingsmassage sollten nur speziell für die Säuglingspflege ausgewiesene Öle verwendet werden. Speiseöle sind nicht geeignet, da diese reizende Bestandteile enthalten können. Paraffinöle eignen sich besonders für empfindliche und zu Allergien neigende Säuglingshaut.

\section{Hautschutz}

Öle können die Haut durch ihre Fähigkeit schützen, wasserlösliche Stoffe abzuweisen, z. B. Urin im Windelbereich. Hinzu kommt der teilokklusive, vor Wasserverlust schützende Effekt.

Differenzierung und Empfehlungen: Bei der Verwendung von Pflanzenölen können Fettsäuren und mögliche zusätzliche Inhaltsstoffe wie Phytosterine und Carotinoide die Hautschutzbarriere stärken.

FAZIT

Evidenzbasierte Empfehlungen für die Anwendung von Ölen in der Säuglingspflege

- Bei leichter, zeitweiliger Hauttrockenheit können speziell für die Bedürfnisse von Säuglingshaut entwickelte Pflegeöle dünn auf die Haut aufgetragen werden.

- Wissenschaftlicher Hintergrund: Öle von pharmazeutischer Qualität, sowohl pflanzlicher als auch mineralischer Herkunft, helfen, Feuchtigkeit in der Haut zu speichern, und unterstützen so die Barrierefunktion der Haut.

- Haushaltsöle sollten bei der Pflege von Säuglingen nicht verwendet werden. Wissenschaftlicher Hintergrund: Haushaltsöle können für die Hautpflege unerwünschte Bestandteile enthalten und ihre Eigenschaften z. B. unter Lichteinfluss verändern. Sie unterliegen weder den Vorgaben der Kosmetikverordnung noch der des Deutschen Arzneibuchs.

- Pflanzenöle mit hohem Ölsäureanteil (zum Beispiel einige Olivenölsorten) können sogar einen schädigenden Einfluss auf die Hautbarriere ausüben.

\section{Inhaltsstoffe von Reinigungs- und Pflegeprodukten für die Säuglingspflege}

Immer wieder wird über Inhaltsstoffe in kosmetischen Produkten diskutiert. Häufig kommt es hierbei auf die persönliche Perspektive an, ob eher pflanzliche oder synthetische Stoffe bevorzugt werden. Auf jeden Fall sollte genauer hingesehen werden, sobald es um die Vermeidung allergener Stoffe geht.

Die Inhaltsstoffangaben auf Produktverpackungen orientieren sich an der Internationalen Nomenklatur für kosmetische Inhaltsstoffe (INCI). Dies ist eine internationale Richtlinie, die insbesondere Allergikern eine Hilfestellung bei der Identifikation von für sie bedenklichen Stoffen geben soll. Bei der Erläuterung der folgenden Inhaltsund Hilfsstoffe haben wir Beispiele von gängigen Stoffen mit ihrer INCl-Bezeichnung aufgeführt.

Kosmetika enthalten unter anderem Grundstoffe und Hilfsstoffe. Diese sorgen zum Beispiel für optimale Konsistenz oder Haltbarkeit. Wir erklären die gängigsten Stoffgruppen und beispielhafte Inhaltsstoffe innerhalb dieser Gruppen.

\section{Spezielle und besondere Inhaltsstoffe}

\section{Calendula}

Die Blüten der Calendula officinalis (Ringelblume) enthalten unter anderem ätherische Öle, Carotinoide und Flavonoide. Calendula-Extrakt hat beruhigende Eigenschaften. Calendula ist häufig in Pflegeprodukten für trockene und rissige Haut oder auch in Sonnenschutzcreme enthalten.

Vorsicht: Bei besonders empfindlicher Säuglingshaut kann Calendula Kontaktekzeme auslösen.

$\rightarrow$ INCl-Bezeichnung: Calendula Officinalis

Kamille

Die Kamille enthält unter anderem Bisabolol, welches als hautberuhigend gilt. Der Inhaltsstoff der echten Kamille (Chamomilla recutita), die am häufigsten in der Kosmetik eingesetzt wird, zeichnet sich durch seine hohe Milde und Verträglichkeit aus.

Reinigungs-, Pflege- und Sonnenschutzprodukte enthalten oft Kamille. Auch für Haarpflegemittel oder entspannende Bade- und Massageöle wird der Inhaltsstoff eingesetzt. In sehr seltenen Fällen kann die Anwendung von Kamille zu Allergien führen (Schleimhautschwellungen und Kontaktekzem).

$\rightarrow$ INCl-Bezeichnung: Chamomilla Recutita 


\section{Zink}

Zink ist ein unentbehrliches Spurenelement für den menschlichen Stoffwechsel. In Babypflegeprodukten kommt es meist als Zink-Sauerstoff-Verbindung (Zinkoxid) vor. Der Inhaltsstoff hilft, schädigende Einwirkungen auf die Haut durch äußere Einflüsse zu vermeiden und reizbare Haut zu beruhigen. Er wirkt antiseptisch. Zinkoxidhaltige Cremes werden häufig als Schutzcreme im Windelbereich verwendet.

$\rightarrow$ INCl-Bezeichnung: Zinc Oxide

\section{Panthenol}

Panthenol (meist Dexpanthenol) ist ein in der Regel synthetischer Inhaltsstoff, der im Körper zu dem Vitamin Pantothensäure umgewandelt wird. Dexpanthenol beruhigt die Haut und unterstützt die Bildung neuer Hautzellen. Der Inhaltsstoff lindert durch Hauttrockenheit bedingten Juckreiz und erhöht sowie stabilisiert den hauteigenen natürlichen Feuchtigkeitshaushalt. Aufgrund seiner beruhigenden Wirkung wird Panthenol häufig in Wundschutzcremes für den sensiblen Windelbereich verwendet.

$\rightarrow$ INCl-Bezeichnung: Panthenol

\section{Grundstoffe}

\section{Öle}

Die Gruppe der Öle teilt sich in Pflanzen- und Mineralbeziehungsweise Paraffinöle auf: Beide helfen, Feuchtigkeit länger in der Haut zu speichern, und beugen so erhöhtem Wasserverlust vor. Eingesetzt werden sie in der Hautpflege und Massage sowie als Zusatzstoffe in Lotionen und Cremes oder auch zur Reinigung, z. B. im Windelbereich.

Sowohl Paraffin- als auch Pflanzenöle ziehen nur in die obersten Hautzellschichten des Stratum corneum ein und wirken dort teilokklusiv, d. h., sie können den Feuchtigkeitsverlust der Haut reduzieren und gleichzeitig die Haut „atmen“ lassen. Sie verstopfen die Hautporen also nicht.

Pflanzenöle werden aus Samen, Blüten oder Blättern gewonnen. Sie besitzen zum Teil Inhaltsstoffe, welche die Hautschutzbarriere stärken können. So enthält Nachtkerzenöl Gamma-Linolensäure, eine Fettsäure, die - in die Lipidmembranen eingebaut-der Haut hilft, ihren Feuchtigkeitsgehalt zu regulieren. Auch Mandelöl ist reich an ungesättigten Fettsäuren sowie an Vitamin $\mathrm{E}$. Es gilt als reizlindernd und feuchtigkeitsspendend, kann aber Allergien auslösen. 
Paraffinöle (auch Mineralöle genannt) basieren im ursprünglichen Sinne auf Pflanzen und Meeresorganismen. In komplexen Verfahren werden hoch gereinigte, schadstofffreie, farb- und geruchlose Paraffine gewonnen. Sie sind frei von allergenen Stoffen, etwa Proteinen, und daher sehr verträglich.

Haushaltsöle, etwa Oliven- oder Sonnenblumenöl, eignen sich nicht für die Babypflege, da sie eine undefinierte, schwankende Qualität besitzen und nicht dermatologisch geprüft sind.

$\rightarrow$ INCl-Bezeichnungen (Beispiele): Simmondsia Chinensis Oil (Jojobaöl), Glycine Soja Oil (Sojaöl), Helianthus Annuus Seed Oil (Sonnenblumenöl), Prunus Amygdalus Dulcis Oil (Mandelöl), Oenothera Biennis Oil (Nachtkerzenöl), Olea Europaea Oil (Olivenöl), Paraffinum Liquidum (Paraffinöl), Elaeis Guineensis Oil (Palmöl)

\section{Ölkomponenten (Emollients)}

Ölkomponenten erfüllen ähnlich wie reine Öle den Zweck, Feuchtigkeit in der Haut zu halten und eine Emulsion reichhaltiger zu gestalten, d. h., den Pflegeeffekt zu erhöhen. Dazu gehören beispielsweise Lipide, Fettalkohole, Fettsäuren und deren Ester (Mono-, Di- und Triglyceride), Phospholipide, Wachse und Silikone.

$\rightarrow$ INCl-Bezeichnungen (Beispiele): Cetyl Alcohol, Ethylhexyl Stearate, Squalan, Dimethicone, Cyclopentasiloxane, Isopropyl Palmitate, C12-15 Alkyl Benzoate, Lanolin

\section{Feuchthaltemittel (Humectants)}

Feuchthaltemittel speichern Wasser in der Haut beziehungsweise helfen, Feuchtigkeit auf der Haut zu halten.

$\rightarrow$ INCl-Bezeichnungen (Beispiele): Glycerin, Urea (Harnstoff), Sorbitol, Propylene Glycol (Propylenglykol), Lactic Acid (Milchsäure)

\section{Grenzflächenaktive Substanzen}

Grenzflächenaktive Substanzen setzen die Oberflächenspannung einer Flüssigkeit herab und ermöglichen so die Durchmischung von Substanzen. Je nach Zusammensetzung und Anwendung bezeichnet man sie auch als Tenside oder Emulgatoren.

\section{Tenside}

Unter Tensiden versteht man waschaktive Substanzen. Sie sind notwendig, um die Haut von fettlöslichen Substanzen zu reinigen, was mit Wasser alleine nur schwer möglich wäre.

$\rightarrow$ INCl-Bezeichnungen (Beispiele): Coca Glucoside, Glyceryl Oleate, Cocamidopropyl Betaine, Sodium Lauroamphoacetate, Sodium Laureth Sulfate.

\section{Emulgatoren}

Emulgatoren sind die wahrscheinlich größte und vielseitigste Stoffgruppe in der Kosmetik. Durch die Herabsetzung der Oberflächenspannung ermöglichen sie die Verbindung zweier nicht mischbarer Flüssigkeiten zu einer Emulsion (Öl und Wasser). In der Kosmetik sind sie unerlässlich, um Fett- und Wasserphase für Lotionen und Cremes zu mischen. Mit ihrer Hilfe werden sowohl Öl-inWasser- (höherer Wasseranteil) als auch Wasser-in-ÖlEmulsionen (höherer Fettanteil) hergestellt.

- Eine Öl-in-Wasser-Emulsion (0/W) zieht rasch in die Haut ein, hinterlässt kaum einen Fettfilm und ist mit Wasser leicht zu entfernen. Produkte mit dieser Emulsionsform werden meist zur Pflege normaler Haut eingesetzt.

- Eine Wasser-in-Öl-Emulsion (W/ 0) hinterlässt einen leichten Fettfilm. Wasser perlt von der eingecremten Haut ab. Produkte mit dieser Emulsionsform werden meist zur Pflege trockener Haut eingesetzt. Die Gruppe der Emulgatoren ist in mehrere Klassen unterteilt: Unter anderem gibt es Zuckeremulgatoren, PEG-Emulgatoren, Fettalkohole, Silikonemulgatoren, Polyglycerin-Emulgatoren.

$\rightarrow$ INCi-Bezeichnungen (Beispiele): Hydrogenated Castor Oil, Polyglyceryl-4 Isostearate, Lecithin, Hydrogenated Palm Glycerides, Potassium Cetyl Phosphate, Polysorbate 20

\section{Sheabutter}

Sheabutter, aus den Nüssen des afrikanischen Sheabaums gewonnen, besitzt eine hohe rückfettende Wirkung. Sie enthält wasserunlösliche Stoffe wie Vitamin E, die dafür sorgen, dass die Haut die Fettsäuren der Sheabutter gut aufnimmt, weich und geschmeidig bleibt.

Sheabutter ist aufgrund ihrer streichfähigen Konsistenz Grundlage vieler Cremes und Lotionen. Sie eignet sich besonders zur Pflege trockener, empfindlicher und reizbarer Haut.

$\rightarrow$ INCl-Bezeichnung: Butyrospermum Parkii Butter

\section{Wollfett}

Das Wollfett (Wollwachs / Lanolin) wird aus der Wolle von Schafen gewonnen und anschließend gereinigt. Das wachsartige Fett kann bis zum Dreifachen seines Gewichts an Wasser binden und ist den körpereigenen Hautfetten ähnlich. Hochreines Lanolin ist sehr gut verträglich und pflegt die Haut geschmeidig, ohne sie auszutrocknen. Lanolin ist als Grundlage für Salben geschätzt, weil es ihnen eine geschmeidige Konsistenz verleiht.

Vorsicht: Lanolin kann, je nach Reinheitsgrad, geringe Spuren von Lanolinalkohol enthalten, der Allergien auslösen kann.

$\rightarrow$ INCl-Bezeichnung: Lanolin 
INFO

Galenik (Zusammensetzung) von Pflegeprodukten Je nach Darreichungsform von Pflegeprodukten sind die Fett- bzw. Wasserphasen von Emulsionen unterschiedlich gewichtet:

- Salbe: Gemisch aus überwiegend Fettstoffen und ge-ringem Wasseranteil

- Creme: Gemisch aus Fett- und Wasserphasen mit hö-herem Fettanteil

- Lotion: Gemisch aus Fett- und höherem Wasseranteil

\section{Hilfsstoffe}

\section{Konservierungsmittel}

Konservierungsmittel werden in der Regel benötigt, sobald ein Produkt Wasser enthält. In Wasser können sich Mikroorganismen vermehren. Die Kontamination eines Hautpflegeprodukts durch solche Mikroorganismen, z. B. Bakterien, Hefe- oder Schimmelpilze, kann zu einer gesundheitlichen Gefährdung des Benutzers führen. Deshalb sind Konservierungsmittel unverzichtbar für die Produktsicherheit. Sie verhindern sowohl das Eindringen als auch die Vermehrung von Mikroorganismen in einem Produkt-von der Herstellung über die Lagerung bis hin zur Verwendung.

Die Gruppe der Konservierungsstoffe ist in mehrere Klassen unterteilt. Unter anderem gibt es halogenorganische Verbindungen, Parabene, Formaldehydabspalter und organische Säuren. Im Gegensatz zu Parabenen, Sodium Benzoaten und Potassium Sorbaten können sowohl halogenorganische Verbindungen (diese enthalten Chlor oder Jod) als auch Formaldehydabspalter und Phenoxyethanol häufiger Kontaktsensibilisierungen bewirken.

$\rightarrow$ INCl-Bezeichnungen (Beispiele): Phenoxyethanol, Methylparaben, Ethylparaben, Sodium Benzoate, Potassium Sorbate

\section{Duftstoffe}

Duftstoffe dienen sowohl in der Tier- als auch in der Pflanzenwelt zur Kommunikation. Zudem sind sie beim Menschen stark mit Erinnerungen verbunden. Bei Säuglingspflegeprodukten wirken sie positiv in Verbindung mit Pflegeritualen zur Vertiefung der Eltern-Kind-Bindung und zur Stärkung der sensorischen Wahrnehmung.

Angenehme Gerüche ziehen an, unangenehme stoßen ab. So werden Kosmetikprodukte häufig mit Düften aufgewertet. Auch lässt sich mit Duftstoffen der manchmal unangenehme Eigengeruch einer Rezeptur ausgleichen. Hierzu können sowohl synthetische als auch natürliche Duftstoffe (z. B. ätherische Öle) eingesetzt werden.

Meist werden Duftstoffe kombiniert und in der Inhaltsstoffangabe als „Parfum“ zusammengefasst. Über diesen
Sammelbegriff wird häufig diskutiert, weil solche Zusammensetzungen mitunter Duftstoffe enthalten, die Allergien auslösen können. Seit 2005 müssen daher die 26 gängigsten allergieverdächtigen Duftstoffe nach Europäischer Kosmetikverordnung in der Inhaltsstoffangabe separat aufgelistet werden.

$\rightarrow$ INCi-Bezeichnungen (Beispiele): Parfum; zu den gängigsten allergieverdächtigen Duftstoffen gehören z.B.: Geraniol, Linalool, Citral, Benzyl Alcohol, Isoeugenol

\section{Antioxidanzien}

Antioxidanzien werden eingesetzt, um zu verhindern, dass im Produkt enthaltene Öle oder Fette vorzeitig oxidieren und somit ranzig werden.

$\rightarrow$ INCl-Bezeichnungen (Beispiele): Tocopherol (Vitamin E), Ascorbyl Palmitate, Tocopheryl Acetate, BHT

\section{Weitere Hilfsstoffe}

Für die pH-Justierung: Zitronensäure, Milchsäure, Natriumhydroxid.

$\rightarrow$ INCl-Bezeichnungen (Beispiele): Citric Acid, Lactic Acid, Sodium Hydroxide

Für die Viskositätseinstellung: Salze, Cellulosen, Polymere

$\rightarrow \quad \mathrm{INCl}$-Bezeichnungen (Beispiele): Sodium Chloride, Hydroxyethyl Cellulose, Carbomer, PEG-150 Distearate, Hydroxypropyl Starch Phosphate

FAZIT

Empfehlungen zur Beschaffenheit von Reinigungs- und Pflegeprodukten für die Säuglingshautpflege

Die Vielfalt der erhältlichen Babypflegeprodukte und ihre unterschiedliche Ausprägung und Zusammensetzung machen ein näheres Eingehen auf einzelne Produkteigenschaften im Rahmen dieses Fachartikels nahezu unmöglich. Auch gibt es für unterschiedliche Konsistenzen, z. B. Gel, Lotion, Milch, Creme usw., keine eindeutig festgelegten differenzierenden Standards. Jedoch lassen sich einige Grundaussagen machen, die auf alle Babypflegeprodukte zutreffen.

- Die Formulierung von Reinigungs- und Pflegeprodukten sollte einen pH-Wert der Haut um 5,5 erhalten.

- Die Formulierung von Reinigungs- und Pflegeprodukten sollte weder die Flora normaler Haut beeinflussen noch die Entwicklung der Säuglingshaut stören.

- Formulierungen sollten keine Irritationen der Säuglingshaut oder der Augen verursachen. 
Autorinnen / Autoren

Ulla Busmann ist Dipl.-Pflegepädagogin und stellvertretende Schulleiterin an der Kath. Schule für Pflegeberufe Essen $\mathrm{gGmbH}$ in Essen.

Claudia Dachs, M.A., Pflegepädagogin, Hebamme, Schulleitung der Berufsfachschule für Hebammen und Entbindungspfleger am Klinikum Augsburg.

Prof. Dr. Hans F. Merk ist Dermatologe in Aachen und war mehr als 20 Jahre lang als Direktor der Aachener Hautklinik tätig.

\section{Korrespondenzadresse}

Univ.-Prof. Dr. med. Hans F. Merk

Direktor (em.) Klinik für Dermatologie \& Allergologie

RWTH Aachen University

E-Mail: hans.merk@post.rwth-aachen.de

\section{Über diesen Artikel}

Die dreiteilige Artikel-Serie zur Pflege der Haut von Neugeborenen und Säuglingen basiert auf dem Handbuch für die Hebammenpraxis „Evidenzbasierte Pflege der Säuglingshaut“"von Claudia Dachs, Ulla Busmann und Prof. Dr. Hans F. Merk, Stuttgart 2016, Thieme

\section{Literatur}

[1] Ananthapadmanabhan KP, Moore DJ, Subramanyan K et al. Cleansing without compromise: the impact of cleansers on the skin barrier and the technology of mild cleansing. Dermatol Ther 2004; 17 (Suppl 1); 16-25

[2] Carpenter P, Richards K. Olive versus mineral oil. Community Pract 2011; 84: 40-42

[3] Chenj, Sadakata M, Ishida M et al. Babymassage ameliorates neonatal jaundice in full-term newborn infants. Tohoku J Exp Med 2011; 223: 97-102
[4] Danby SG, Al Enezi T, Sultan A et al. Effect of olive and sunflower seed oil on the adult skin barrier: implications for neonatal skin care. Pediatr Dermatol 2013; 30: 42-50

[5] Danby SG, Bedwell C, Cork M. Neonatal Skin Care and Toxicology. In: Eichenfield LF, Frieden IJ, Mathes EF, Zaenglein AL, eds. Neonatal and Infant Dermatology, 3 rd ed. Philadelphia: Saunders; 2015: 46-56. ISBN 978-1-4557-2638-7

[6] Danby SG, Cork M. The Skin Barrier in Atopic Dermatitis, Ch. 27. In: Irvine A, Hoeger P, Yan A, eds. Harper's Textbook of Pediatric Dermatology. Vol. 1, 3rd ed. Chichester, UK: WileyBlackwell Publishing Ltd.; 2011. ISBN 978-1-4051-7695-8

[7] Field T, Cullen C, Largie $S$ et al. Lavender bath oil reduces stress and crying and enhances sleep in very young infants. Early Hum Dev 2008; 84: 399-401

[8] Kulkarni A, Kaushik JS, Gupta P et al. Massage and touch therapy in neonates: the current evidence. Indian Pediatr 2010; 47: 771-776

[9] Patzelt A, Lademann J, Richter H, Darvin M, Schanzer S, Thiede G, Sterry W, Vergou T, Hauser M. In vivo investigations on the penetration of various oils and their influence on the skin barrier. Skin Res Technol 2012; 18: 364-369

[10] Rawlings AV, Lombard KJ. A review on the extensive skin benefits of mineral oil. Int J Cosmet Sci 2012; 34: 511-518

[11] Serrano MS, Doren FM, Wilson L. Teaching Chilean mothers to massage their full-term infants: effects an maternal breastfeeding and infant weight gain at age 2 and 4 months. J Perinat Neonatal Nurs 2010; 24: 172-181

[12] Stamatas GN et al. Lipid uptake and skin occlusion following topical application of oils on adult and infant skin. J Derm Sci 2008; 50: 135-142

[13] Walker P. Olive oil versus rnedicinal grade mineral oil for baby massage. Community Pract 2010; 83: 20; author reply

\section{Bibliografie}

DOI https://doi.org/10.1055/a-0729-5326

Die Hebamme 2018; 31: 324-330

(c) Georg Thieme Verlag KG Stuttgart · New York ISSN 0932-8122 